\title{
Nanofibrillar cellulose hydrogels and reconstructed hydrogels as matrices for controlled drug release
}

\section{Paukkonen, Heli}

2017

Paukkonen, H , Kunnari, M , Lauren , P , Hakkarainen , T, Auvinen , V-V , Oksanen , T , Koivuniemi , R , Yliperttula , M \& Laaksonen , T 2017 , ' Nanofibrillar cellulose hydrogels and reconstructed hydrogels as matrices for controlled drug release ' , International Journal of Pharmaceutics, vol. 532 , no. 1 , pp. 269-280 . https://doi.org/10.1016/j.ijpharm.2017.09.002

http://hdl.handle.net/10138/297937

https://doi.org/10.1016/j.jpharm.2017.09.002

cc_by_nc_nd

acceptedVersion

Downloaded from Helda, University of Helsinki institutional repository.

This is an electronic reprint of the original article.

This reprint may differ from the original in pagination and typographic detail.

Please cite the original version. 
Nanofibrillar cellulose hydrogels and reconstructed hydrogels as matrices for controlled drug release

\title{
Heli Paukkonen ${ }^{a}$, Mikko Kunnaria ${ }^{\text {, Patrick Lauréna }}$, Tiina Hakkarainena, Vili-Veli Auvinen ${ }^{\mathbf{a}, \mathrm{b}}$,
}

\section{, Timo Oksanen ${ }^{a}$, Raili Koivuniemia ${ }^{a}$, Marjo Yliperttulaa,c, Timo Laaksonen ${ }^{a, b}$}

${ }^{a}$ Division of Pharmaceutical Biosciences, Faculty of Pharmacy, University of Helsinki, P.O. Box 56, FI-00014 Helsinki, Finland

${ }^{\mathrm{b}}$ Department of Chemistry and Bioengineering, Tampere University of Technology, P.O. Box 541, FI-33101 Tampere, Finland

${ }^{c}$ Department of Pharmaceutical and Pharmacological Sciences, via Marzalo 5, University of Padova, Padova, Italy

\begin{abstract}
Concentrated $3 \%$ and $6.5 \%$ anionic nanofibrillar cellulose (ANFC) hydrogels were introduced as matrix reservoirs for controlled delivery applications of small molecules and proteins. A further aim was to study how the freeze-drying and subsequent rehydration of ANFC hydrogel affects the rheological properties and drug release of selected model compounds from the reconstructed hydrogels. It was demonstrated that the $3 \%$ and $6.5 \%$ ANFC hydrogels can be freeze-dried with suitable excipients into highly porous aerogel structures and redispersed back into the hydrogel form without significant change in the rheological properties. Freeze-drying did not affect the drug release properties from redispersed ANFC hydrogels, indicating that these systems could be stored in the dry form and only redispersed when needed. For large molecules, the diffusion coefficients were significantly smaller when higher ANFC fiber content was used, indicating that the amount of ANFC fibers in the hydrogel can be used to control the release rate. The release of small molecules was controlled with the ANFC fiber content only to a moderate extent. The results indicate that ANFC hydrogel can be used for controlled delivery of several types of molecules and that the hydrogel can be successfully freeze-dried and redispersed.
\end{abstract}

Keywords: Nanofibrillar cellulose, drug release, diffusion, hydrogel, aerogel, freeze-drying, rheology

Abbreviations: ANFC, Anionic nanofibrillar cellulose; BSA, bovine serum albumin; $\boldsymbol{D}$, diffusion coefficient; DSC, differential scanning calorimetry; exp, excipients trehalose and polyethylene glycol; FITC-DEX, fluorescein isothiocyanate-dextran; KETO, ketoprofen; LZ, lysozyme; MZ, metronidazole; NAD, nadolol; NFC, nanofibrillar cellulose; PEG, polyethylene glycol; TEMPO, [(2,2,6,6-Tetramethylpiperidin-1-yl)oxyl]; tre, trehalose 


\section{Introduction}

Hydrogels have various uses in biomedical applications and are well suited for regenerative medicine (Peppas, et al. 2006; Slaughter, et al. 2009) and controlled drug delivery (Gupta, et al. 2002). Nanofibrillated cellulose (NFC) hydrogel has been widely investigated in pharmaceutical and biomedical fields, such as in drug delivery (Laurén, et al. 2014), scaffold synthesis (Borges, et al. 2011), and as cell and drug carriers (Thérien-Aubin, et al. 2016; Valo, et al. 2013). NFC hydrogel is also applicable as a cell culture scaffold for three dimensional cell culturing (Bhattacharya, et al. 2012; Lou, et al. 2014; Lou, et al. 2015; Malinen, et al. 2014). It is produced from the natural biopolymer cellulose, and wood pulp is a commonly used starting material in the production (Klemm, et al. 2011). The surface of native NFC fibers can be further chemically modified with e.g. TEMPO [(2,2,6,6-Tetramethylpiperidin-1-yl)oxyl] oxidation to contain negatively charged carboxyl groups directly on the fiber surface (Saito, et al. 2006; Saito, et al. 2007). This way, it is possible to produce anionic NFC (ANFC) fibers. Further, native as well as anionic TEMPO oxidized NFC grades have been shown to be biocompatible and nontoxic in various in vitro cell models (Alexandrescu, et al. 2013; Bhattacharya, et al. 2012; Hannukainen, et al. 2012; Hua, et al. 2014; Vartiainen, et al. 2011), making them appealing for pharmaceutical applications.

Since cellulose retains moisture and is both biocompatible (in humans) and biodegradable (in nature), NFC has been investigated in wound treatment applications facing the need for faster and more effective wound healing (Chinga-Carrasco and Syverud 2014; Lin and Dufresne 2014; Powell, et al. 2016; Zhang, et al. 2013). Previously, we have indicated the suitability of native NFC wound dressing in clinical use in treatment of skin graft donor sites of burn patients (Hakkarainen, et al. 2016). NFC hydrogels are also of special interest in the wound healing applications due to high moisture retaining ability and biocompatibility (Lin and Dufresne 2014; Zhang, et al. 2013). Furthermore, NFC hydrogel can be freeze-dried into solid aerogel structure, which may be utilized in drug delivery (Pääkkö, et al. 2008; Valo, et al. 2013). However, it is generally acknowledged that the drying of NFC promotes irreversible hydrogen bonding between neighboring NFC nanofibrils, known as hornification (Diniz, et al. 2004; Wan, et al. 2010). So far it has been a major manufacturing challenge to produce dry NFC while maintaining the nano-scale dimensions, and several techniques such as oven drying, freezedrying, supercritical drying and spray-drying have been investigated (Kolakovic, et al. 2012; Peng, et al. 2012; Peng, et al. 2013). Freeze-drying produces NFC aerogels with lamellar structures and significant lamellar aggregation (Peng, et al. 2012).

In addition to the preservation of NFC nano-scale dimensions throughout drying process, the preservation of rheological properties of the NFC hydrogels is important for drug delivery 
applications. These should stay reasonably similar during processing in order to have reliable, controlled and predictable drug release formulations. It has been shown that once-dried TEMPOoxidized ANFC fibers can be re-dispersed, reversing the hornification effect introduced by the drying, by involving higher energy consumption (Kekäläinen, et al. 2014). This is probably due to the high surface charge density present in the TEMPO-oxidized ANFC fibers, which is much lower in the absence of carboxyl groups on the native NFC fiber surface. Furthermore, the electrostatic repulsion forces working between the anionically-charged fibers stabilizes the aqueous suspension (Isogai, et al. 2011). The high surface density of negative charges has been used to electrostatically immobilize biomolecules (Weishaupt, et al. 2015). Therefore, the high aspect ratio as well as anionic charge of ANFC fibers can be utilized as rate-controlling parameters in controlled drug release applications. Rheological properties and redispersability of highly concentrated $3-6.5 \%$ ANFC hydrogels after drying and consequent rehydration and re-gelling have not been studied extensively in the literature, even though these might be especially useful in e.g. wound healing applications. Previous studies have mainly focused on the evaluation of redispersibility and rheological properties of dilute $0.5-1 \%$ NFC suspensions after spray drying or freeze-drying (Missoum, et al. 2012; Žepič, et al. 2014).

Freeze-drying is often used in the manufacturing of solid dosage forms of sensitive protein pharmaceuticals to improve the storage stability (Carpenter, et al. 1997; Tang and Pikal 2004). However, cryo- and lyoprotectants are usually required to prevent structural damage of protein pharmaceuticals induced by freezing and drying steps in the freeze-drying process (Carpenter, et al. 1997; Franks 1998; Hubálek 2003). We studied the prospect that these excipients could retain the structure of ANFC to a certain extent during freeze-drying. Furthermore, based on the literature (Kekäläinen, et al. 2014), ANFC seems to be a more suitable grade than native NFC for the preparation of freeze-dried formulations intended for consequent reconstruction and rehydration, and was chosen as the hydrogel forming material.

We propose that with regard to preservation of ANFC structure it may be beneficial to use trehalose to replace the hydrogens bonds that are lost due to the sublimation of water in the freeze drying of ANFC. The suitability of trehalose as a lyo- and cryoprotectant has already been confirmed in the freeze-drying of protein pharmaceuticals (Chang, et al. 2005; Jovanović, et al. 2006), red blood cells (Han, et al. 2005), platelets (Crowe, et al. 2003; Wolkers, et al. 2001) and liposomes (Christensen, et al. 2007). It has been proposed that the molecular mechanism of protein as well as cell membrane stabilization with trehalose is achieved through water-trehalose hydrogen-bond replacement, coating by a trapped water layer and/or mechanical inhibition of the conformational fluctuations (Lins, et al. 2004). Low trehalose concentration of 5-100 $\mathrm{mM}$ does not provide cryoprotection for proteins, but in 
combination with $1 \%$ polyethylene glycol (PEG) stabilization can be achieved (Carpenter, et al. 1993; Prestrelski, et al. 1993). Furthermore, PEG as hygroscopic polymer improves the water uptake (Jeon, et al. 1991) which can be beneficial for reconstitution and rehydration of ANFC aerogels.

The aim of the current work was to characterize rheological as well as drug release properties of $3 \%$ and $6.5 \%$ ANFC hydrogels prior to and after freeze-drying and reconstruction. Here, a combination of trehalose and PEG 6000 was chosen as the cryo- and lyoprotectants in the freeze-drying of ANFC hydrogels into aerogels in order to minimize the hornification of ANFC fibers and to preserve the nano-scale structure of ANFC fibers. The freeze-dried aerogel formulations were rehydrated and regelled into their original concentrations in order to reform the hydrogel structure prior to the release testing and rheological measurements. Model compounds in the release studies included small molecules metronidazole (MZ), nadolol (NAD) and ketoprofen (KETO) with molecular weight below 500 M. 4 kDa FITC-dextran (FITC-DEX), lysozyme (LZ) and bovine serum albumin (BSA) represented high molecular weight compounds. The model compounds were selected based on their size, wieght and different charges at $\mathrm{pH} 7$.

The main release mechanism of small molecules and proteins from hydrogels can be characterized by Fickian diffusion (Censi, et al. 2012), as the ANFC hydrogel does not exhibit swelling or any other internal or external triggers for the release in PBS buffer solutions. The diffusion rate of solutes may be influenced through weak interactions such as electrostatic, hydrophobic and hydrogen bonding between the hydrogel polymer network and solutes. The permeation of small molecules and proteins through hydrogels is further affected by their size, shape (Hoffman 2012) and the availability of free water molecules to hydrate and dissolve the solute molecules in addition to hydrogel pore size, pore size distribution and the pore interconnections affect the diffusion rate of molecules through the hydrogel. It is therefore clear that the hydrogel structure plays an important role for the release rate and must be rigorously controlled.

\section{Materials and methods}

\subsection{Materials}

$3.2 \%$ (11804-3) and $6.8 \%$ (11815-3) anionic NFC (ANFC) hydrogels (FibDex ${ }^{\mathrm{TM}}$ ) were kindly provided by UPM-Kymmene Corporation, Finland. Cellulose kraft pulp was chemically modified and fibrillated to form ANFC hydrogels (Saito, et al. 2006; Saito, et al. 2007). A Carboxylic acid content 1,06 mmol / g pulp was determined by conductometric titration according to the standard SCAN-CM 65:02. The diameter of most of the fibrils is in the range of 4-10 nm and length 500 - 10 $000 \mathrm{~nm}$ measured using electron microscopies. All model compounds and reagents were of analytical 
grade. Bio-Rad Protein Assay reagent was purchased from Bio-Rad, USA. 4 and 10 kDa FITCdextran molecules were purchased from Sigma-Aldrich, Sweden. D-(+)-trehalose dihydrate was purchased from Sigma-Aldrich, USA. Metronidazole was purchased from Sigma-Aldrich, China. Nadolol was purchased from Sigma-Aldrich, Finland. Ketoprofen was purchased from Orion Pharma, Finland. Lysozyme from hen egg white was purchased from Roche, Germany. Polyethylene glycol 6000 was purchased from Fluka, Switzerland. Dulbecco's Phosphate Buffered Saline (10X) concentrate without calcium and magnesium was purchased from Gibco, UK. Acetonitrile was of analytical grade, Sigma-Aldrich, Germany.

\subsection{Methods}

\subsubsection{Preparation of the ANFC hydrogel formulations}

ANFC hydrogel formulations were prepared in $10 \mathrm{ml}$ syringes. The hydrogels were homogenized with model compounds by mixing for 10 min inside two attached syringes (200 times through the syringe nozzle). Table 1 contains the formulation compositions of the physical mixtures of ANFC hydrogels and model compounds. The ANFC amount in the final formulations was $3 \%$ or $6.5 \%$ $(\mathrm{m} / \mathrm{m})$. The measured $\mathrm{pH}$ for pure ANFC hydrogels was 7. For MZ, NAD and KETO, an excess amount of each drug was used in relation to their solubility in $\mathrm{pH}$ 7. Therefore, the drug containing ANFC hydrogel formulations were monolithic dispersions. MZ, NAD and KETO were added as dry powders into the ANFC hydrogels with final concentrations of $2 \%$ for MZ, $1.7 \%$ for NAD and 3.4 $\%$ for KETO. The amount of $4 \mathrm{kDa}$ FITC-DEX, BSA and LZ in the formulations did not exceed the solubility limit at $\mathrm{pH}$ 7. Therefore, these formulations were considered to be monolithic solutions. 4 $\mathrm{kDa}$ FITC-DEX was added into the ANFC hydrogel from $1 \mathrm{mg} / \mathrm{ml}$ stock solution with a $1 \%$ final concentration of FITC-DEX. BSA and LZ were added as dry powders into the ANFC hydrogels with final concentrations of $1 \%$ for BSA and $0.5 \%$ for LZ. The $3 \%$ and $6.5 \%$ ANFC hydrogel formulations with MZ, NAD, KETO and BSA were also prepared with $1 \%$ of PEG6000 and $0.3 \%$

of trehalose for freeze-drying. Dilute $1.1 \%$ ANFC hydrogels were crosslinked with cations calcium $\left(\mathrm{Ca}^{2+)}\right.$, aluminum $\left(\mathrm{Al}^{3+}\right)$ and iron $\left(\mathrm{Fe}^{3+}\right)$ and were used to study the effect of crosslinking on rheology and drug release properties with $10 \mathrm{kDa}$ FITC-DEX and MZ (detailed information in supplement). 
Table 1. Physicochemical properties of the model compounds and the formulation compositions with $3 \%$ and $6.5 \%$ ANFC hydrogels. ${ }^{*} \mathrm{MZ}, \mathrm{NAD}, \mathrm{KETO}$ and BSA formulations were also prepared with $1 \%$ of PEG6000 and $0.3 \%$ of trehalose for freeze-drying.

\begin{tabular}{|c|c|c|c|c|c|c|c|}
\hline Compound & $\begin{array}{c}\text { MW } \\
(\mathrm{g} / \mathrm{mol})\end{array}$ & $\begin{array}{l}\text { Solubility } \\
(\mathrm{mg} / \mathrm{ml})\end{array}$ & $\begin{array}{c}\text { pKa } \\
/ \mathbf{p I}\end{array}$ & $\begin{array}{c}\text { Charge } \\
\text { at } \\
\text { pH } 7.0\end{array}$ & $\begin{array}{c}\text { Hydrodynamic } \\
\text { diameter } \\
(\mathrm{nm})\end{array}$ & $\begin{array}{c}\text { Weight \% } \\
(\mathrm{m} / \mathrm{m}) \\
\text { in hydrogel }\end{array}$ & Ref. \\
\hline KETO* & 254 & $\begin{array}{l}0.107\left(25^{\circ} \mathrm{C}\right) \\
\sim 40.7(\mathrm{pH} 7) \dagger\end{array}$ & 4.06 & - & & 3.4 & $\begin{array}{l}\text { (Avdeef, et al. 2000; Sheng, et } \\
\text { al. 2006; Singhai, et al. 1996; } \\
\text { Zhou, et al. 2005) }\end{array}$ \\
\hline NAD* & 309 & $\begin{array}{l}8.3\left(25^{\circ} \mathrm{C}\right) \\
8.3(\mathrm{pH} 7)\end{array}$ & 9.28 & + & & 1.7 & $\begin{array}{l}\text { (Avdeef and Berger 2001; } \\
\text { Zhou, et al. 2005) }\end{array}$ \\
\hline MZ* & 171 & $\begin{array}{c}10.5\left(25^{\circ} \mathrm{C}\right) \\
10.5(\mathrm{pH} 7)\end{array}$ & 2.38 & $\varnothing$ & & 2 & $\begin{array}{l}\text { (Kim, et al. 2012; Wu and } \\
\text { Fassihi 2005; Zhou, et al. } \\
\text { 2005) }\end{array}$ \\
\hline $\mathrm{BSA}^{*}$ & $6.65 \times 10^{4}$ & 40 & $\begin{array}{c}4.6- \\
4.8\end{array}$ & - & $\sim 7$ & 1 & $\begin{array}{l}\text { (Sigma-Aldrich; Yohannes, et } \\
\text { al. 2010) }\end{array}$ \\
\hline $\mathrm{LZ}$ & $1.47 \times 10^{4}$ & $>10$ & 11.1 & + & $\sim 3.7$ & 0.5 & $\begin{array}{l}\text { (Fritz, et al. 1995; Szymańska } \\
\text { and Ślósarek 2012) }\end{array}$ \\
\hline FITC-DEX & 4000 & 50 & & $\varnothing$ & $\begin{array}{l}2.8 \\
\sim 2\end{array}$ & 1 & (Sigma-Aldrich) \\
\hline
\end{tabular}

$\uparrow 40.7 \mathrm{mg} / \mathrm{ml}$ solubility at $\mathrm{pH} 7$ was used for the calculation of diffusion coefficients of KETO with the Hiquchi equation. Excess KETO remained in the crystalline form in the hydrogel at 3.4\%, and therefore in practice initial drug concentration $>$ drug solubility.

$1.1 \%(\mathrm{w} / \mathrm{v})$ anionic ANFC hydrogel (UPM Oyj, Finland) was used with crosslinking cations aluminum sulfate hydrate (Sigma-Aldrich, USA), iron(III) nitrate nonahydrate (Sigma-Aldrich, Germany) and calcium chloride (Sigma-Aldrich, Japan). Hydrogels were crosslinked by weighing and dissolving the solid powder with ANFC and left to set for 48 hours before release studies. Final concentrations of crosslinking cations $\mathrm{Al}^{3+}, \mathrm{Fe}^{3+}$ and $\mathrm{Ca}^{2+}$ used with ANFC were $2.5 \mathrm{mmol} / \mathrm{kg}, 2.2$ $\mathrm{mmol} / \mathrm{kg}$ and $4.4 \mathrm{mmol} / \mathrm{kg}$ respectively. Release study model compounds MZ and $10 \mathrm{kDa}$ FITC-DEX were dissolved with crosslinked and non-crosslinked ANFC to a final amount of $2 \%$ wt and $0.6 \%$ wt of hydrogel respectively.

\subsubsection{Freeze-drying protocol}

Pure ANFC hydrogels, ANFC hydrogels with 1\% of PEG6000 and $0.3 \%$ of trehalose as well as ANFC hydrogels with MZ, NAD, KETO, BSA together with the cryoprotectants were used to produce aerogels with the freeze-drying process. $2.5 \mathrm{ml}$ of each sample was placed inside a $10 \mathrm{ml}$ syringe, followed by fast freezing by dipping the hydrogel in liquid nitrogen for 1 minute. The frozen samples were immediately transferred to a freeze dryer (FreeZone 2.5, LabConco, USA) and freezedried in a vacuum (70 mTorr) at a sublimating temperature of $-52{ }^{\circ} \mathrm{C}$ for $29 \mathrm{~h}$. The final freeze dried samples were sealed in the syringes and stored inside a silica desiccator until used. Freeze-dried ANFC aerogels with MZ, NAD, KETO and BSA were rehydrated with ultrapure water into their original concentrations gravimetrically. The aerogels were then redispersed into a hydrogel form by homogenization inside two syringes for $10 \mathrm{~min}$ (200 times through syringe nozzle). 


\subsubsection{Scanning electron microscopy (SEM)}

The morphology of the freeze-dried aerogels was imaged with a scanning electron microscope Quanta FEG250 (SEM, FEI Company, USA). The aerogels were fractured manually for analysis of the inner aerogel structure or cut with a scalpel for the analysis of the aerogel surface structure. Micrographs of the cross-sections as well as surface structures of the aerogels were obtained. Before imaging the samples were fixed onto a two-sided carbon tape and sputtered with platinum for 25s with an Agar sputter device (Agar Scientific Ltd., UK).

\subsubsection{Thermogravimetry analysis (TGA)}

The residual moisture content of the aerogels was determined using TGA (TGA 850, Mettler-Toledo, Switzerland). Samples were heated from 25 to $240^{\circ} \mathrm{C}$ at a heating rate of $10^{\circ} \mathrm{C} / \mathrm{min}$ in nitrogen $(40$ $\mathrm{ml} / \mathrm{min}$ ) atmosphere. Residual moisture content of the aerogels was determined as a mass loss (\%) of evaporated water. TGA data was gathered from single measurement.

\subsubsection{Differential scanning calorimetry (DSC)}

Thermal analysis of the freeze dried ANFC aerogels were carried out using a differential scanning calorimeter Mettler Toledo DSC 823e (Mettler Toledo, Giessen, Germany). The samples were placed in sealed aluminum pans with closed lids and heated at a scanning rate of $10{ }^{\circ} \mathrm{C} / \mathrm{min}$ between 25 and $200{ }^{\circ} \mathrm{C}$ in nitrogen atmosphere. The data was analyzed with STARe software (Mettler-Toledo, Giessen, Germany). All DSC measurements were performed after one month storage period inside a silica desiccator at $20^{\circ} \mathrm{C}$. DSC data was gathered from single measurement.

\subsubsection{Rheological measurements}

The rheological measurements were performed at $37^{\circ} \mathrm{C}$ with HAAKE Viscotester iQ Rheometer (Thermo Fisher Scientific, Karlsruhe, Germany) equipped with a Peltier system for temperature control. Results were analyzed with HAAKE RheoWin 4.0 software (Thermo Fisher Scientific). Parallel $35 \mathrm{~mm}$ diameter steel plate-and-plate geometry was used with a $1 \mathrm{~mm}$ gap in all measurements. Before each measurement, the samples were allowed to rest for $5 \mathrm{~min}$ at $37^{\circ} \mathrm{C}$. Controlled stress amplitude sweeps were performed to determine the linear viscoelastic region for different ANFC hydrogel formulations. Constant angular frequency $\omega=1 \mathrm{~Hz}$ and oscillatory stress between $1^{*} 10^{-4}-500 \mathrm{~Pa}$ was used in all amplitude sweeps. The chosen oscillatory stresses for frequency sweeps were $\tau=20 \mathrm{~Pa}$ (crosslinked and non-crosslinked $1.1 \%$ ANFC hydrogels), $\tau=50$ $\mathrm{Pa}(3 \%$ ANFC hydrogel) and $\tau=100 \mathrm{~Pa}(6.5 \%$ ANFC hydrogel) and the angular frequency range 
was 0.6 - 125.7 rads $^{-1}$. Shear viscosity was measured by increasing the shear rate from 0.1 to 1000 1/s. All measurements were performed in triplicate.

\subsubsection{In vitro release studies}

Disc molds were filled with $1.07 \mathrm{~g}$ of ANFC hydrogel formulations. The mods had a constant flat surface area of $1.33 \mathrm{~cm}^{2}$ exposed to the release buffer. Discs were placed in $150 \mathrm{ml}$ amber glass vessels on top of a holder. The vessels were filled to a final volume of $70 \mathrm{ml}$ with $\mathrm{pH} 7.4$ phosphate buffered saline (1 x DPBS) and kept at $37^{\circ} \mathrm{C}$ under constant magnetic stirring (400 rpm) on top of a multi-position magnetic stirrer IKA RT10 (IKA-Werke GmbH \& Co KG, Germany). 1.5 ml samples were collected from the vessels and replaced with fresh buffer up to $144 \mathrm{~h}$. All model compounds were found to be stable under the experimental test conditions. All experiments were done in triplicate.

Established mathematical equations that take into consideration the structure of the device, the ratio of initial drug concentration to drug solubility and the device geometry were used to quantitatively describe the diffusion controlled drug release (Siepmann and Siepmann 2012). The diffusion coefficients were calculated with Higuchi equation (1) for small molecules with a presumption that these systems could be described as monolithic dispersions (initial drug concentration $>$ drug solubility) with slab geometry (Higuchi 1963; Higuchi 1961; Siepmann and Peppas 2011; Thakkar, et al. 2009):

$Q=\sqrt{D\left(2 C_{\text {ini }}-C_{s}\right) C_{s} t}$

$D=\frac{Q^{2}}{2 C_{i n i} C_{s} t}$

where $Q$ is the amount of drug released in time $t$ per unit area, $D$ is the diffusion coefficient of the drug within the matrix system, $C_{i n i}$ represents the initial drug concentration in the matrix system, $C_{s}$ is the drug solubility in the matrix media. Solubility values at $\mathrm{pH} 7$ were used for KETO, NAD and $\mathrm{MZ}$ as an estimate of $C_{s}$ in the ANFC hydrogel matrix. For the calculation of diffusion coefficient the equation can be reduced to (2) as in our system $C_{i n i}>C_{s}$.

Diffusion coefficients for large molecules $\left(M_{w} \geq 1 \mathrm{kDa}\right)$ were calculated with the unsteady-state form of Fick's second law of diffusion (3) with early values of time when $0<M_{t} / M_{\infty}<0.6$ (Siepmann and Siepmann 2012). The assumption was that these systems could be described as monolithic solutions (initial drug concentration $<$ drug solubility) with slab geometry: 
$\frac{M_{t}}{M_{\infty}}=4\left(\frac{D t}{\pi L^{2}}\right)^{\frac{1}{2}}$

where $M_{t}$ and $M_{\infty}$ denote the cumulative amounts of drug released at time $\mathrm{t}$ and infinite time $\infty ; D$ is the diffusion coefficient of the drug within the matrix system; $L$ represents the thickness $(1 \mathrm{~cm})$ of the hydrogel. $D$ was obtained from fitting the data of $M_{t} / M_{\infty}$ at selected time points.

\subsubsection{Quantification of model compounds from in vitro release samples}

KETO and NAD concentrations from the in vitro release samples were analyzed with Ultra performance liquid chromatography (UPLC) instrument Acquity UPLC (Waters, USA). For KETO, the used column was HSS-C18 $1.8 \mu \mathrm{m}(2.1 \times 50 \mathrm{~mm})$ (Waters, USA) at $30^{\circ} \mathrm{C}$. The flow rate was 0.5 $\mathrm{ml} / \mathrm{min}$ and the injection volume $5 \mu \mathrm{l}$. The KETO detection was performed at $255 \mathrm{~nm}$ wavelength. During the gradient run, the mobile phase consisted of a mixture of acetonitrile and $15 \mathrm{mM}$ phosphate buffer $\mathrm{pH} 2$ in 25:75 ratio at 0-3 min. After $3 \mathrm{~min}$, the mobile phase composition was changed to 75:25 ratio. The retention time of KETO was $1.69 \mathrm{~min}$. The linear concentration of KETO was established in the range of $0.1-25 \mu \mathrm{g} / \mathrm{ml}$ and LOQ for KETO was $0.03 \mu \mathrm{g} / \mathrm{ml}$. For NAD, the used column was HSS-T3 $1.8 \mu \mathrm{m}(2.1 \times 50 \mathrm{~mm})$ (Waters,USA) at $30^{\circ} \mathrm{C}$. The flow rate was $0.5 \mathrm{ml} / \mathrm{min}$ and the injection volume $5 \mu$ l. The NAD detection was performed at $215 \mathrm{~nm}$ wavelength. During the gradient run the mobile phase consisted of a mixture of acetonitrile and $15 \mathrm{mM}$ phosphate buffer $\mathrm{pH}$ 2 in 10:90 ratio at 0-3 min. After 3 min the mobile phase composition was changed to 50:50 ratio. The retention time of NAD was $0.92 \mathrm{~min}$. The linear concentration of NAD was established in the range of $0.1-50 \mu \mathrm{g} / \mathrm{ml}$ and LOQ for NAD was $0.1 \mu \mathrm{g} / \mathrm{ml}$.

FITC-DEX quantification was performed by fluorescence intensity measurements with an automated multimode plate reader Varioskan Flash (Thermo Fisher Scientific, Finland). $200 \mu 1$ of samples were pipetted into wells of 96-well OptiPlate plates (PerkinElmer, Finland). Excitation wavelength 490 $\mathrm{nm}$, emission wavelength $520 \mathrm{~nm}$ and $12 \mathrm{~nm}$ bandwidth were used. The established linear range for FITC-DEX calibration curves was $0.005-0.15 \mu \mathrm{g} / \mathrm{ml}$ with squared correlation coefficients above 0.99 $(\mathrm{R}>0.99)$.

MZ and LZ quantification was performed by spectrophotometric analysis with Cary $100 \mathrm{UV}-\mathrm{Vis}$ spectrophotometer (Varian Inc., USA). For MZ, absorbance was measured in $1 \mathrm{~cm}$ cell at $320 \mathrm{~nm}$ with $12 \mathrm{~nm}$ bandwidth against blank. The established linear range for MZ calibration curve was 3 $35 \mu \mathrm{g} / \mathrm{ml}$ with squared correlation coefficient above $0.99(\mathrm{R}>0.99)$. For LZ, absorbance was 
measured in $1 \mathrm{~cm}$ cell at $280 \mathrm{~nm}$ with $12 \mathrm{~nm}$ bandwidth against blank. The established linear range for $\mathrm{LZ}$ calibration curve was 1 - $17 \mu \mathrm{g} / \mathrm{ml}$ with squared correlation coefficient above $0.99(\mathrm{R}>0.99)$.

BSA quantification was performed by colorimetric Bio-Rad protein assay, which is based on Bradford dye-binding method (Bradford 1976; González-González, et al. 2011). Shortly, $150 \mu 1$ of each BSA standard sample and BSA sample was pipetted in triplicate into separate microtiter well plates and $50 \mu \mathrm{l}$ of diluted Bradford dye reagent was added. Sample and reagent were mixed thoroughly with a multi-channel pipet and incubated for 20 minutes at $20{ }^{\circ} \mathrm{C}$ before absorbance measurement. Absorbance was measured with a well plate reader Varioskan Flash ((Thermo Fisher Scientific, Finland) at $470 \mathrm{~nm}$ and $595 \mathrm{~nm}$ with $5 \mathrm{~nm}$ bandwidth. The established linear range for BSA calibration curves was 1 - $40 \mu \mathrm{g} / \mathrm{ml}$ with squared correlation coefficients above $0.99(\mathrm{R}>0.99)$. Linearization of the assay was used to improve accuracy and sensitivity (Ernst and Zor 2010; Zor and Selinger 1996).

\section{Results and discussion}

\subsection{Morphology of ANFC aerogels}

The ANFC hydrogel formulations were rapidly frozen with liquid nitrogen prior to freeze-drying. Fast supercooling with liquid nitrogen typically produces a large number of small ice crystals (Tang and Pikal 2004). It has been reported that the NFC aerogel structure formation is directly related to the size and distribution of ice crystals in the frozen state (Aulin, et al. 2010). Furthermore, the extent of protonation on the surface carboxyl groups of ANFC produced by TEMPO oxidation affects the aerogel structure (Jiang and Hsieh 2016). High protonation reportedly results in the formation of a porous ultrathin lamellar aerogel structures and high water uptake upon rehydration. PEG6000 present in the aerogels likely improved the wettability and re-gelling of the aerogels as it is generally recognized as a hygroscopic polymer that improves the water uptake (Jeon, et al. 1991).

The highly porous aerogel structure of freeze-dried ANFC was confirmed with SEM micrographs (Fig. 1). All of the ANFC aerogels with and without encapsulated model compounds exhibited high porosity and similar morphology. The drug molecules did not affect the ANFC aerogel structure significantly (Fig. S1). Rather similar aerogel structures were observed for both the $3 \%$ and $6.5 \%$ ANFC aerogels. We can speculate that the rapid liquid nitrogen freezing prior to freeze-drying, as well as the high content of entangled ANFC fibers in the $3 \%$ and $6.5 \%$ ANFC hydrogels, aided in the preservation of the entangled ANFC fiber structure in the aerogels to some extent. Furthermore, trehalose and PEG 6000 were used as lyoprotectants to preserve the structure of the ANFC aerogels. The conclusion is supported by previous studies where these excipients have shown remarkable 
synergistic capacity to retain the structure and activity of proteins during freeze-drying (Carpenter, et al. 1993; Prestrelski, et al. 1993). Although the morphology of the aerogels was rather similar, differences were observed in the rheological properties before and after freeze-drying.

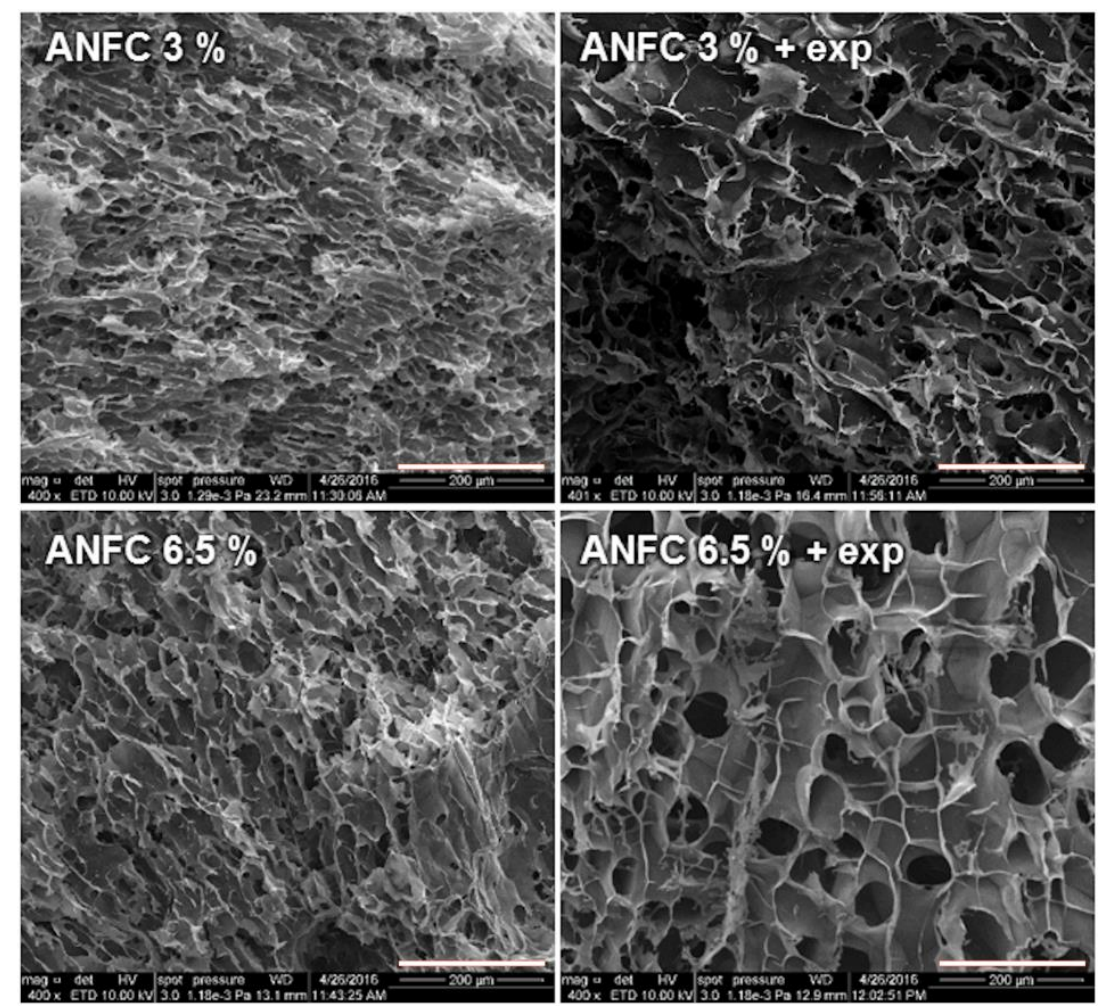

Figure 1. SEM micrographs of freeze-dried highly porous ANFC aerogels. 3\% and 6.5\% ANFC hydrogels were freeze-dried without (left) and with the excipients (right). Scale bar is $200 \mu \mathrm{m}$. Abbreviation exp refers to $0.3 \%$ trehalose and $1 \%$ PEG 6000.

\subsection{Thermogravimetry analysis (TGA) and differential scanning calorimetry (DSC)}

Residual moisture content of the aerogels was determined with TGA and mass change (\%) for all formulations is presented in Table 2. The aerogel produced from $6.5 \%$ ANFC hydrogel contained $7.5 \%$ of residual moisture, while the aerogel produced from $3 \%$ ANFC hydrogel contained $6.8 \%$ of residual moisture. As there are more fibers in the more concentrated ANFC aerogel, there is also more tightly bound surface water resulting in slightly higher residual moisture content when the same processing parameters were used for both aerogels. When trehalose and PEG were used with ANFC, the corresponding residual moisture was decreased for both $3 \%$ and $6.5 \%$ ANFC aerogels. In previous studies trehalose and PEG have been used to protect proteins from undergoing conformational charges during freeze-drying due to favorable interactions with PEG (Mi, et al. 2004) and trehalose (Chang, et al. 2005; Jovanović, et al. 2006). One of the speculated stabilization mechanisms of trehalose in the literature is the replacement of water molecules hydrogen bonded to polar groups on the surface of materials during freeze-drying (Lins, et al. 2004; Wolkers, et al. 2002). 
Due to this mechanism the amount of tightly bound surface water on ANFC fibers is reduced and lower residual moisture content could be observed when trehalose and PEG were used in the formulations.

Table 2. TGA and DSC analysis of different ANFC aerogel formulations after freeze-drying $(n=1)$. Residual moisture content of the aerogels was determined as a mass loss (\%) of evaporated water. Melting points of model compounds are reported as onset temperatures. All formulations except pure $3 \%$ and $6.5 \%$ ANFC contained $1 \%$ PEG $6000 / 0.3 \%$ trehalose in the final aerogel. Abbreviation exp refers to $1 \%$ PEG $6000 / 0.3 \%$ trehalose.

\begin{tabular}{|c|c|c|}
\hline Formulation & Water content (\%) & $\operatorname{Tm}\left({ }^{\circ} \mathrm{C}\right)$ for drug or ANFC \\
\hline ANFC $3 \%$ & 6.77 & 171.5 \\
\hline ANFC $3 \%+\exp$ & 4.11 & 170 (ANFC/trehalose) \\
\hline ANFC $6.5 \%$ & 7.48 & 174.0 \\
\hline ANFC $6.5 \%+\exp$ & 5.81 & 187 (ANFC/trehalose) \\
\hline BSA $1 \% /$ ANFC $3 \%$ & 4.79 & $\mathrm{nd}$ \\
\hline BSA $1 \% /$ ANFC $6.5 \%$ & 6.05 & nd \\
\hline MZ $100 \%$ reference & nd & 159.4 \\
\hline MZ $2 \% /$ ANFC $3 \%$ & 2.42 & 148.7 \\
\hline MZ $2 \%$ / ANFC $6.5 \%$ & 4.98 & 143.5 \\
\hline NAD $100 \%$ reference & nd & 128.8 \\
\hline NAD $1.7 \%$ / ANFC $3 \%$ & 2.90 & 127.3 \\
\hline NAD $1.7 \% /$ ANFC $6.5 \%$ & 4.79 & 128.3 \\
\hline KETO $100 \%$ reference & nd & 94.6 \\
\hline KETO $3.4 \%$ / ANFC $3 \%$ & 1.96 & 81 \\
\hline KETO $3.4 \%$ / ANFC $6.5 \%$ & 3.84 & 84 \\
\hline
\end{tabular}

For drug containing aerogels, the hydrophilicity (Table 1 in materials and methods) of the model compounds affected the residual moisture content of the aerogels in addition to ANFC fiber amount. The residual moisture content was higher for formulations with BSA, NAD and MZ, whereas KETO as the most hydrophobic compound tested had the lowest values. All model compounds decreased the moisture content when compared to the plain hydrogels. It might be possible to optimize the freeze-drying process for constant residual moisture content, but this was not seen as necessary here, as the main goal was to later redisperse the aerogels into hydrogels. In future studies more research will be focused on the duration of primary drying and the secondary drying steps that removes the tightly bound surface water. However, the low residual moisture content indicated that the sublimation of water was effective and the process parameters were suitable for the aerogel production.

The DSC thermograms of aerogels showed a reduction in the melting points of model compounds with regard to references (Table 2). It has been shown in earlier studies that the melting peaks may disappear from DSC thermograms if the drug and polymer matrix are well integrated. Valo et al. have previously shown that the NFC grade and source affect the magnitude of drug interactions in freeze- 
dried cellulose formulations (Valo, et al. 2013). Therefore, the observed differences in the melting points of model compounds with regard to pure references indicate interactions between the ANFC fibers and the model compounds. It has been shown in a previous study that the binding of drugs into NFC fibers is $\mathrm{pH}$ dependent and the electrostatic forces are the main binding mechanism thus resulting in the strongest binding between cationic molecules and anionic NFC fibers (Kolakovic et al., 2013). Furthermore, it has been suggested that $\pi$ stacking and hydrogen bonding may take place as binding mechanisms in addition to electrostatic interactions. The structure of NFC and ANFC is rather similar and therefore the $\mathrm{pH}$ dependent electrostatic binding of molecules into ANFC fibers was expected to be the main interaction for binding of positively charged model compounds. For negatively charged and neutral model compounds, we expected to observe only minimal binding with ANFC fibers. The in vitro release studies were performed in order to evaluate more specifically the effect of molecule charge on the release profiles.

\subsection{Rheology}

The numerous microscopic pores in the aerogel structure caused by the sublimated ice crystals ensured enhanced water penetration into the aerogel structure upon rehydration (Jiang and Hsieh 2016). Furthermore, ANFC is known to have high water absorption and retention capacity (Jiang and Hsieh 2014). The rehydration and hydrogel formation was further enhanced by mechanical mixing (Figure S1). However, freeze-drying of plain $3 \%$ and $6.5 \%$ ANFC without excipients reduced the viscosity values of redispersed gels significantly (Fig. 2). Both grades showed $\sim 150 \%$ higher viscosities before freeze-drying than their respective rehydrated samples at shear rates $0.1-40(1 / \mathrm{s})$. The presence of excipients significantly reduced the gap to $\sim 30 \%$ by increasing the viscosity of the freeze-dried samples and slightly lowering the plain hydrogel viscosities. This was more notable for the $6.5 \%$ hydrogels. The slight decrease in viscosity with ANFC hydrogels with excipients, when compared to plain hydrogels without excipients, was probably due to the stabilization effect of lowering the surface tension of the system (Gilányi, et al. 2006). Therefore, the excipients themselves had minimal impact in the overall viscosity of the system. However, the main difference between the rehydrated hydrogels (with vs. without excipients) was most likely due to larger amount of aggregation in the samples dried and rehydrated without excipients. Therefore, the increase in viscosity of the rehydrated samples with the excipients was attributed to the preservation of structural integrity, limiting the formation of aggregates. The addition of model compounds did not significantly change the viscosity when compared to the ANFC hydrogels with excipients, regardless of the excessive (i.e. undissolved) amount of MZ, KETO and NAD. For BSA and MZ however, the gap between the viscosities was slightly wider prior to and after freeze-drying than with the ANFC 
hydrogels containing only excipients. The gap was narrower for NAD and KETO. All samples expressed a steady decrease in viscosity with increasing shear rate. Shear thinning has been reported earlier on strong NFC hydrogels, and therefore ANFC hydrogel with similar shear thinning behaviour is considered to be a pseudoplastic material (Pääkkö, et al. 2007). Freeze-drying did not have an effect on these properties after rehydration, and the results indicate structural similarities before and after freeze-drying processes. 



Figure 2. Shear rate viscosity of plain ANFC ( $3 \%$ and $6.5 \%$ ) as well as different ANFC hydrogel formulations before and after freeze-drying and redispersion (mean \pm S.D., $n=3$ ). ANFC hydrogels contained 1\% BSA, 2\% metronidazole (MZ), $3.4 \%$ ketoprofen (KETO) or $1.7 \%$ NAD (NAD). Abbreviations: FD $=$ freeze-drying, $\exp =$ trehalose, PEG6000. 
The effect of freeze-drying on the viscoelastic properties of ANFC hydrogels with and without excipients was studied. Freeze-drying lowered the storage modulus ( $G^{\prime}$ ) values of all samples with and without excipients (Fig. 3); however, the gap between the G' values of the non-freeze-dried hydrogels and freeze-dried (i.e. rehydrated) hydrogels becomes significantly narrower with excipients. Therefore, the G' values of rehydrated ANFC hydrogel samples with excipients resembled the original values of the hydrogels without excipients before freeze-drying on both grades ( $3 \%$ and $6.5 \%$ ). All samples, including mixtures with model compounds (Fig. S3, S4), were relatively independent of the angular frequency at investigated ranges. Additionally, G' values were higher than loss modulus (G') values, which indicates an elastic behavior in strong ANFC hydrogels (Pääkkö, et al. 2007). Therefore, the elastic properties of hydrogels remained after freeze-drying, indicating structural similarities as was shown with viscosity measurements.
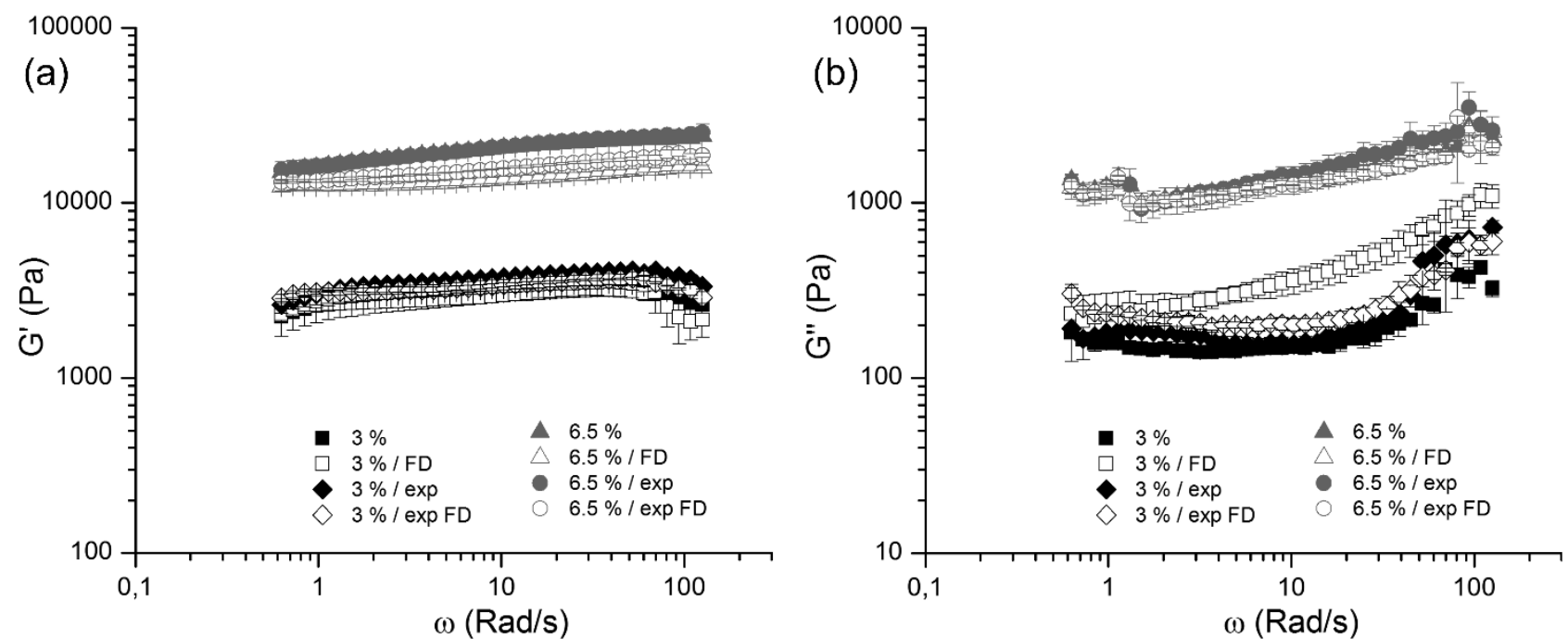

Figure 3. Storage $\left(G^{\prime}\right)$ and loss modulus $\left(G^{\prime}\right.$ ') $)$ of ANFC hydrogels before and after freeze-drying and redispersion (mean \pm S.D., $n=3$ ). Abbreviations: $F D=$ freeze-drying, exp $=$ trehalose, PEG6000.

A slight increase in G' can be observed with the stronger $6.5 \%$ hydrogels towards higher frequencies. For the less viscous $3 \%$ ANFC the increase was not as prominent. Similarly, the G', values increased towards higher frequencies on both grades of ANFC hydrogels; however, the effect of freeze-drying varies. In contrast to $6.5 \%$ ANFC hydrogel the G' of $3 \%$ the freeze-dried ANFC samples had higher values than the non-freeze-dried hydrogels, especially with the samples not containing excipients. The G' of $6.5 \%$ rehydrated hydrogels was slightly lower in comparison to non-freeze-dried samples (as was observed with G'). However, the presence of excipients had a significant impact in reducing the effects of freeze-drying in both concentrations of rehydrated hydrogels. Therefore, it can be concluded that at higher frequencies, the excipients attributed to the elastic properties of ANFC hydrogel after rehydration, i.e. with the absence of excipients, G', increases while G' decreases and 
the viscous characteristics become more predominant over the elastic properties (Dimić-Mišić, et al. 2013).

Based on the results of rheological measurements it was evident that the rheological properties were preserved to a greater extent when excipients were used as stabilizers during freeze-drying. In dilution studies of the re-gelled ANFC hydrogels, we observed that freeze-dried ANFC with excipients remained suspended without aggregates (data not shown). On the contrary, visible aggregates were observed when ANFC hydrogel was freeze-dried without excipients followed by re-gelling and dilution. This may further indicate that the excipients reduced the irreversible hydrogen bonding between the ANFC fibers in hydrogel as the freeze-dried ANFC fibers with excipients remained hydrated more effectively.

Additionally, we investigated the effects of crosslinking cations on the rheological properties of $1.1 \%$ ANFC hydrogel. The ANFC hydrogels were crosslinked with cations $\mathrm{Al}^{3+}, \mathrm{Fe}^{3+}$ or $\mathrm{Ca}^{2+}$. All crosslinked systems showed an increase in viscosity, more notably with the trivalent cations $\mathrm{Al}^{3+}$ and $\mathrm{Fe}^{3+}$ (Fig. S5, top). Similar increase in G' and G', was observed (Fig. S5, bottom). The results indicate a form of stabilization effect as with excipients; however, this was due to a different mechanism of stiffening the hydrogel structure, i.e strengthening the bonds within the hydrophilic segments of the hydrogel. More detailed information can be found within the supplementary material.

\subsection{In vitro drug release}

The in vitro release studies were performed in order the estimate the effect of ANFC fiber concentration as well as freeze-drying on the release profiles of a variety of model compounds from the hydrogels. The ANFC hydrogel matrix did not swell in the PBS buffer used in the release studies thus retaining the original shape and dimensions of the hydrogel matrix throughout the release experiments. Therefore, it was hypothesized that the slower diffusion in the non-swelling hydrogel matrix would control the drug release rates. This property as well as high water retention capacity of ANFC can be utilized beneficially in controlled drug delivery applications.

The $\mathrm{pH}$ of the ANFC hydrogels was 7 prior to the addition of model compounds, and the $\mathrm{pH}$ of the release medium was kept at 7.4 with PBS. Due to moderate buffering capacity of ANFC, the pH of the hydrogel matrix exposed to PBS buffer was estimated to be approximately 7 after the addition of model compounds and excipients. Physical mixtures of KETO, NAD, and MZ with ANFC contained excess amounts of drug in relation to their solubility at $\mathrm{pH} 7$ and were defined as monolithic dispersions (Table 1, Materials and methods). The amount of KETO at $34 \mathrm{mg} / \mathrm{ml}$ in the ANFC 
hydrogels was below the $\mathrm{pH} 7$ solubility limit of $40 \mathrm{mg} / \mathrm{ml}$. However, in practice excess amount of undissolved KETO remained in the ANFC hydrogel matrix resulting in a monolithic dispersion (Fig. S2). $4 \mathrm{kDa}$ FITC-DEX, LZ, and BSA were all completely dissolved in the ANFC hydrogels. By definition these formulations were monolithic solutions.

The addition of trehalose and PEG 6000 lowered the viscosity of $3 \%$ and $6.5 \%$ ANFC hydrogels slightly (Fig. 2 and 3). Therefore, the release studies were performed from plain ANFC hydrogels, hydrogels with excipients and from freeze-dried and reconstructed hydrogels that also contained excipients. The results from the release studies are presented in Fig. 4. The drug release profiles were rather similar from plain 3\% (Fig. 4a) and excipient containing 3\% (Fig. 4b) ANFC hydrogels. Relatively similar drug release profiles were also obtained from plain $6.5 \%$ (Fig. 4c) and excipient containing $6.5 \%$ (Fig. 4d) ANFC hydrogels. It is apparent that the ANFC hydrogel concentration affected the release profiles. However, the release profiles were similar before and after freeze-drying. For small molecules MZ, KETO and NAD the effect of charge seemed to have a weak to moderate impact on the cumulative release profiles in $\mathrm{pH} 7.4$ (Fig. 4). MZ was in the neutral form and had the fastest drug release followed by cationic NAD and anionic KETO. For the relatively hydrophilic neutral MZ and cationic NAD the charge of the molecules may have affected the drug release profiles as MZ diffused faster out from the ANFC hydrogels. The surface of ANFC is negatively charged at pH 7 due to carboxyl groups directly at the fiber surface. It has been previously proposed that electrostatic interactions between chemically modified ANFC fibers and charged molecules can affect the drug release properties (Kolakovic, et al. 2013; Weishaupt, et al. 2015). Therefore, the slower drug release of NAD may have been caused by stronger attractive electrostatic interaction between the surface of ANFC fibers and cationic NAD. MZ as a neutral molecule at $\mathrm{pH} 7$ had likely negligible interactions with ANFC fibers and the release mechanism of MZ was purely affected by the diffusion through the free water phase present in the ANFC hydrogel matrix.

To study the effect of crosslinking of ANFC hydrogel on drug release and rheology (supplementary material, Fig. S5), low concentration (1.1\%) ANFC hydrogels were used to emphasize the effect of $\mathrm{Al}^{3+}, \mathrm{Fe}^{3+}$ or $\mathrm{Ca}^{2+}$ crosslinking cations. The viscosity of the hydrogels was increased by the presence of the crosslinking cations, depending on the cation used $\left(\mathrm{Al}^{3+}>\mathrm{Fe}^{3+}>\mathrm{Ca}^{2+}\right)$. However, crosslinking did not have a significant effect on the drug release profiles with two different model compounds MZ and $10 \mathrm{kDa}$ FITC-DEX (Fig. S6). It was concluded that rheological properties could be altered with cationic crosslinking without affecting drug release rates, as the diffusion seems to occur purely through the water phase of ANFC hydrogels. However, the more concentrated uncrosslinked 3\% and $6.5 \%$ ANFC hydrogels had significantly higher viscosity and ANFC fiber content creating more 
barrier for the diffusion. The release of $\mathrm{MZ}$ reached $100 \%$ after $50 \mathrm{~h}$ from the $1.1 \%$ ANFC hydrogel as well as from $3 \%$ and $6.5 \%$ ANFC hydrogels. The finding supports the conclusion that the diffusion of small molecules was affected by the availability of free water molecules in the 3 and $6.5 \%$ ANFC.
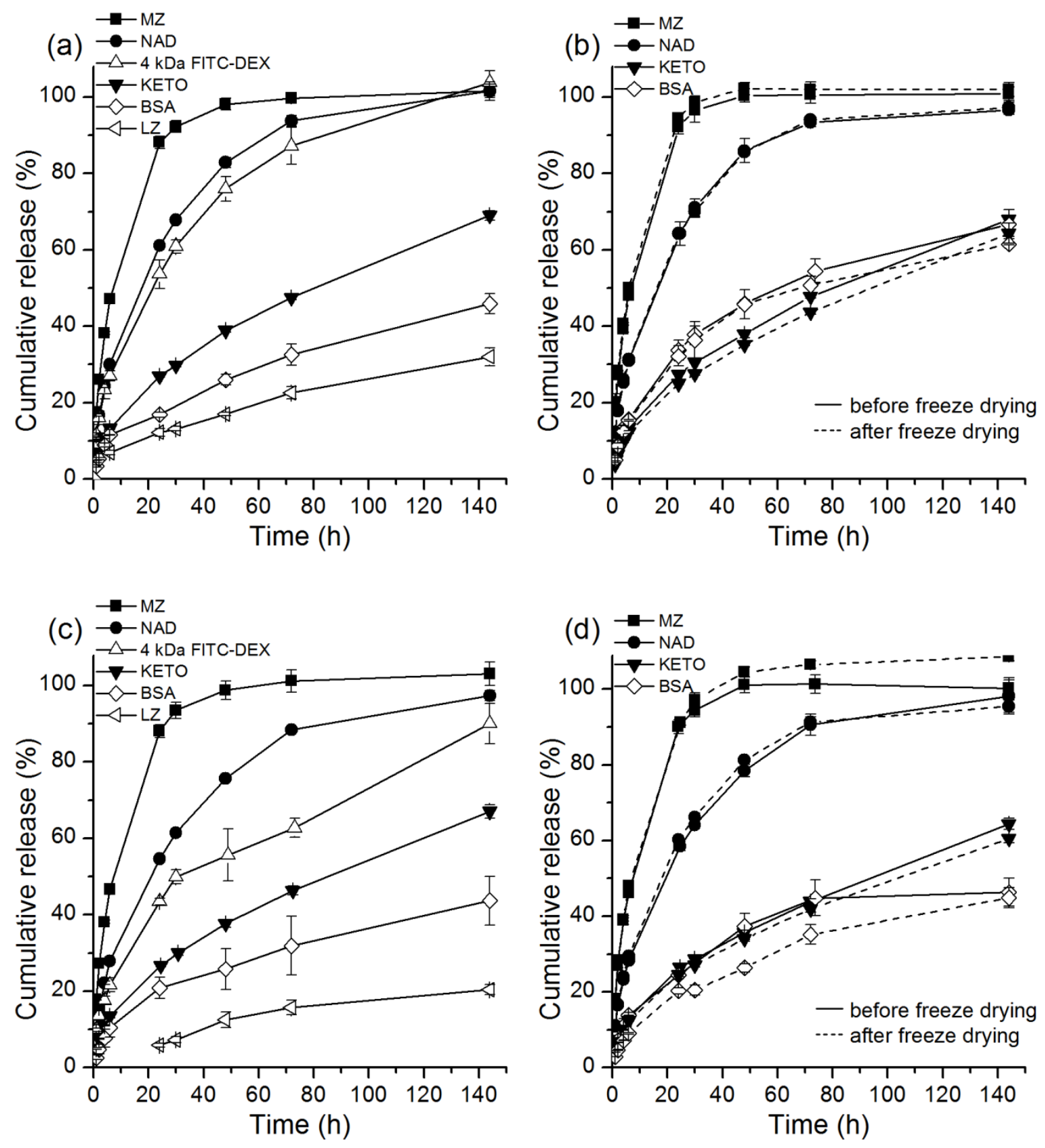

Figure 4. The effect of ANFC hydrogel concentration and freeze-drying on the release profiles of MZ, NAD, KETO, BSA, LZ and 4 kDa FITC-DEX(mean \pm S.D., n=3) . Formulations were based on plain 3\% ANFC hydrogel (a), 3\% ANFC hydrogel with PEG6000 and trehalose before and after freeze-drying (b), plain 6.5\% ANFC hydrogel (c), and 6.5\% ANFC hydrogel with PEG6000 and trehalose before and after freeze-drying (d). Release experiments were performed in PBS ( $\mathrm{pH} 7.4)$ at $37^{\circ} \mathrm{C}$. 
The dissolution rate and the excess amount of the small model compounds in the ANFC hydrogels may have influenced the drug release profiles in addition to the charge of the molecules even though the release experiments were performed under sink conditions. This was observed as prolonged drug release rate for anionic KETO, which based solely on its charge should have diffused faster out from the hydrogel matrix due to electrostatic repulsion with ANFC fibers. Overall, the cumulative release of KETO was below $70 \%$ for all tested hydrogels at day 6, whereas MZ reached $100 \%$ and NAD nearly $100 \%$ cumulative release. The slower drug release rate of KETO may be attributed to amount dependent dissolution rate of KETO inside the ANFC hydrogel matrix as it has been previously reported that the dissolution rate of KETO is strongly dose dependent even under sink conditions (Yazdanian, et al. 2004). Overall, the higher 6.5\% ANFC fiber content reduced the drug release of small molecules only slightly more effectively with regard to corresponding release profiles from 3 $\%$ ANFC hydrogels. Therefore, the amount of ANFC fibers can be used to control the drug release rate of small molecules only to a moderate extent. Furthermore, the freeze-drying did not significantly affect drug release properties of small molecules as the re-gelled formulations exhibited rather similar release behavior as the corresponding pre-freeze-dried formulations (Fig. 4b and 4d). The quality of the ANFC hydrogels as a matrix for controlled drug release applications for small molecules remained unaltered regardless of the drying step as the drug release properties were retained. It was therefore clearly demonstrated that the drug containing ANFC hydrogels can be freeze-dried and re-gelled successfully without any loss on drug release properties.

For large model compounds BSA, LZ, and FITC-DEX the net charge at $\mathrm{pH} 7$, molecular size and ANFC fiber content had a significant impact on the cumulative release profiles (Fig. 4a and 4c). Neutral FITC-DEX had the fastest release rate followed by anionic BSA and cationic LZ. The $4 \mathrm{kDa}$ FITC-DEX reached 100\% release from plain 3\% ANFC hydrogel and 85\% release from plain 6.5\% ANFC hydrogel at day 6. This indicates that the ANFC fiber content did control the release of FITCDEX, but not as effectively as for LZ and BSA. 4 kDa FITC-DEX has significantly smaller size than 14.7 $\mathrm{kDa} \mathrm{LZ}$ and $66.5 \mathrm{kDa} \mathrm{BSA}$ and therefore it was expected that the release of FITC-DEX would be faster. Cationic LZ had the slowest release of approximately 30\% from 3\% ANFC and below 20\% from $6.5 \%$ ANFC hydrogels during 6 days. The anionic BSA had approximately $40 \%$ release from both hydrogel concentrations during 6 days.

It is clear that the cationic charge of LZ reduced the release rate though electrostatic attractive interaction with ANFC fibers. The conclusion is supported by the physicochemical properties of LZ and BSA (Table 1, Materials and methods) as well as similar behavior and slow release of cationic NAD. The molecule size and hydrodynamic diameter of LZ is smaller than for BSA and without any 
electrostatic interaction the release of LZ would have likely been faster. The results indicate that for large molecules (above $14 \mathrm{kDa}$ ) the charge of the molecule was more significant than the molar mass and hydrodynamic diameter in terms of impact on the cumulative release profile.

Furthermore, the cumulative release of none of the large molecules from the ANFC hydrogel matrixes reached $100 \%$. The physical entrapment of these large molecules in the highly entangled ANFC fibers' network likely allowed less free motion for diffusion than for small molecules, which was observed as sustained release for BSA and LZ. BSA was the only large model compound that was freeze-dried with ANFC and excipients. The release profiles of BSA from 3.5\% and $6.5 \%$ ANFC hydrogels were similar before and after freeze-drying (Fig. 4b and 4d) indicating that the release properties of large molecules may be retained even after the drying and re-gelling. This is a highly beneficial property for drug delivery applications of protein pharmaceuticals in addition to small molecules inside a hydrogel matrix as the formulation can be stored at dry state for prolonged storage and rehydrated upon need. For example, the use of ANFC as a hydrogel matrix for the delivery of drugs and growth factors into wound areas could have promoting effects on wound healing by increasing the healing rate and efficacy.

\subsection{The diffusivity in the hydrogel matrix}

For the evaluation of diffusion rate without the impact of solubility, the Higuchi equation (Eq.2, see Materials and Methods) was used to calculate the diffusion coefficients (D) for small molecules (Table 3). We suggest that this is a more suitable way to evaluate the diffusivity of small molecules inside the hydrogels when excess drug with regard to solubility is used in the hydrogel matrix. As described previously literature values of solubility at $\mathrm{pH} 7$ for MZ, NAD and KETO (Table 1) were used for the determination of diffusion coefficients. It should be noted that the $\mathrm{pH} 7$ is a region where the solubility of KETO drastically improves due to ionization and the amount of KETO may affect the dissolution rate (Avdeef, et al. 2000; Yazdanian, et al. 2004). The approximations of diffusion coefficients should be used to compare the D values of small molecules in different hydrogel formulations prior to and after freeze-drying.

The order of the magnitude of diffusion coefficients in all 3\% and $6.5 \%$ ANFC hydrogels was MZ $(\varnothing)>$ NAD $(+)>$ KETO (-). Furthermore, the diffusion coefficients from $3 \%$ ANFC hydrogels were only slightly higher than the corresponding values from $6.5 \%$ hydrogels. This can be explained by the lower viscosity, G' (elastic response) and G' (viscous behavior) of 3\% ANFC hydrogels when compared to the $6.5 \%$ hydrogels (see Fig. 2 and 3). The diffusion coefficients were rather similar for both hydrogels without excipients, with excipients and after freeze-drying. Therefore, freeze-drying 
did not significantly alter the drug release properties of ANFC hydrogels for small molecules. Overall for small molecules, apart from KETO, the addition of excipients into 3\% and 6.5\% ANFC hydrogels slightly increased the diffusion coefficients. This can be explained by the lower viscosity of the excipient containing ANFC hydrogels with regard to pure hydrogels (Fig. 2).

In water, small molecules have typically diffusion coefficients between $600-900 \times 10^{-8} \mathrm{~cm}^{2} / \mathrm{s}$ (Hazel and Sidell 1987). In this regard, both of the ANFC hydrogel concentrations did provide moderate control to the drug release, especially for NAD and KETO as the diffusion coefficients were below $400 \times 10^{-8} \mathrm{~cm}^{2} / \mathrm{s}$ for NAD and $65 \times 10^{-8} \mathrm{~cm}^{2} / \mathrm{s}$ for KETO. This indicates that the diffusion of small molecules with a cationic charge is affected by the negative surface charge of ANFC fibers through electrostatic attractive interactions. The diffusion of KETO may have been affected by hindered dissolution rate of KETO in the ANFC hydrogel matrices and is the reason for low apparent D values of KETO. However, the diffusion coefficients of KETO were almost the same prior to and after freeze-drying, indicating that the freeze-drying process did not affect the drug release properties of ANFC hydrogels. For, MZ the diffusion through the ANFC hydrogel matrices was relatively free resembling the diffusion of small molecules in water as the diffusion coefficients from all hydrogel formulations were above $600 \times 10^{-8} \mathrm{~cm}^{2} / \mathrm{s}$. The experimentally verified release profiles of MZ, NAD and KETO fitted well with theoretical predictions by Higuchi equation (Fig. S7).

Table 3. Diffusion coefficients for model compounds in different ANFC hydrogels. Values are presented for $3 \%$ and $6.5 \%$ ANFC hydrogels with and without excipients. Freeze-dried formulations were re-gelled prior to diffusion studies. The net charge of compounds at $\mathrm{pH} 7$ is in parenthesis. The abbreviation exp refers to PEG 6000 and trehalose.

\begin{tabular}{|c|c|c|c|c|c|c|}
\hline \multirow{2}{*}{ Compound } & \multicolumn{6}{|c|}{ Diffusion coefficients for model compounds in different ANFC hydrogels $\left(10^{-8} \mathrm{~cm}^{2} / \mathrm{s}\right)$} \\
\hline & $3 \%$ ANFC & $\begin{array}{c}3 \% \\
\text { ANFC/exp }\end{array}$ & $\begin{array}{c}\text { FD 3\% } \\
\text { ANFC/exp }\end{array}$ & $6.5 \%$ ANFC & $\begin{array}{c}6.5 \% \\
\text { ANFC/exp }\end{array}$ & $\begin{array}{c}\text { FD } 6.5 \% \\
\text { ANFC/exp }\end{array}$ \\
\hline Ketoprofen (-) & 62,9 & 60,9 & 54,5 & 58,0 & 52,8 & 47,4 \\
\hline NAD (+) & 365,6 & 393,4 & 383,4 & 301,1 & 322,3 & 349,7 \\
\hline Metronidazole (ø) & 745,7 & 823,0 & 841,5 & 733,3 & 761,8 & 779,6 \\
\hline BSA (-) & 7,7 & 23,4 & 22,0 & 7,4 & 15,2 & 9,5 \\
\hline Lysozyme (+) & 4,0 & $n d$ & nd & 2,3 & nd & nd \\
\hline $\begin{array}{l}4 \text { kDa FITC- } \\
\text { dextran (ø) }\end{array}$ & 58,9 & nd & $n d$ & 35,6 & $n d$ & nd \\
\hline
\end{tabular}

The commonly used Fickian model was used to calculate diffusion coefficients inside the hydrogels for large molecules (Eq. 3, see Materials and Methods). Significantly lower diffusion coefficients were observed as opposed to small molecules (Table 3). Therefore, the molecular weight clearly affects the diffusion through the ANFC hydrogel, with a more pronounced restraining effect for larger molecules. The experimentally verified release profiles of 4kDa FITC-DEX, BSA and LZ fitted also 
well with theoretical predictions by Fickian model (Fig. S7). The order of magnitude for diffusion coefficients in all 3\% and $6.5 \%$ ANFC hydrogels was $4 \mathrm{kDa}$ FITC-DEX (ø) $>66.5 \mathrm{kDa}$ BSA (-) $>$ 14.7 kDa LZ (+). For $4 \mathrm{kDa}$ FITC-DEX, the diffusion rate was 1.7 times faster through plain 3\% ANFC hydrogel than through the plain $6.5 \%$ one with diffusion coefficients of 58.9 and $35.6 \times 10^{-8}$ $\mathrm{cm}^{2} / \mathrm{s}$ respectively. For $66.5 \mathrm{kDa}$ BSA the diffusion coefficients were similar in plain $3 \%$ and $6.5 \%$ ANFC hydrogels with values of 7.7 and $7.4 \times 10^{-8} \mathrm{~cm}^{2} / \mathrm{s}$ respectively. The diffusion rate of $14.7 \mathrm{kDa}$ LZ was 1.7 times faster from the plain 3\% ANFC hydrogel than from $6.5 \%$ one with diffusion coefficients of 4.0 and $2.3 \times 10^{-8} \mathrm{~cm}^{2} / \mathrm{s}$ respectively. The cationic charge of LZ prolonged the diffusion through the ANFC fiber network as discussed previously. Similar prolonged release due to electrostatic attractive interaction was also observed for the small molecule model compound NAD. In the literature, solution diffusivity values of $104 \times 10^{-8} \mathrm{x} \mathrm{cm}^{2} \mathrm{~s}-1$ for LZ and $60 \times 10^{-8} \mathrm{~cm}^{2} \mathrm{~s}-1$ for BSA have been reported (Koutsopoulos, et al. 2009; Walters, et al. 1984). The ANFC hydrogels provided a significant control for the diffusion of large molecules as the diffusion coefficients from all hydrogels were significantly lower than the reported literature values in water for LZ and BSA.

The higher diffusion coefficients for large model compounds through the $3 \%$ ANFC hydrogel can be explained by the lower viscosity of $3 \%$ ANFC hydrogel. Overall, the diffusion rate of the anionic $66.5 \mathrm{kDa} \mathrm{BSA}$ was not as slow as for the cationic 14.7 $\mathrm{kDa} \mathrm{LZ}$, but was still slower than for $4 \mathrm{kDa}$ FITC-dextran. BSA has a higher molar mass than LZ and therefore the charge of LZ had a bigger impact on the diffusion rate than the molecular size. It can be concluded that the diffusion of large molecules through ANFC hydrogel is affected by the charge to a greater extent than by the hydrodynamic diameter. Furthermore, the size still affects the diffusion coefficients as the smaller 4 kDa FITC-DEX had higher D values than either BSA or LZ.

BSA was used as a model protein compound in freeze-drying. The diffusion coefficients of BSA were affected by the addition of PEG 6000 and trehalose as well as freeze-drying. The excipients decreased slightly the viscosity of the hydrogels, which may have attributed to the higher diffusion coefficients from hydrogels with excipients. The freeze-drying of 3\% ANFC hydrogel did not significantly alter the release of BSA. By contrast, the diffusion was approximately $40 \%$ slower from the freeze-dried 6.5\% ANFC hydrogel than from the non-freeze-dried sample. NFC is known to undergo irreversible hornification upon dehydration although ANFC is reportedly less affected by this phenomena upon rehydration (Diniz, et al. 2004; Kekäläinen, et al. 2014; Peng, et al. 2012). The structure of the ANFC fibers may have been slightly modified during freeze-drying to an extent that affects the diffusion of large molecules such as BSA. This phenomenon was not observed for any other model compound and suggests that these changes are limited in their scope and occur at length scale larger than the 
small molecules. We conclude that the release properties of all model compounds were preserved in $3 \%$ hydrogels even after freeze-drying. As for the freeze-dried 6.5\% hydrogels, only the release of $66.5 \mathrm{kDa}$ BSA was altered, but the diffusion coefficients were still approximately the same as from plain $6.5 \%$ hydrogel without excipients.

\section{Conclusions}

The chemically modified ANFC hydrogels can be successfully freeze-dried into aerogels and redispersed into the hydrogel form. Cryoprotectants were effectively used to preserve the rheological properties of the rehydrated aerogels and the release profiles of the model compounds were similar before and after freeze-drying. This is a highly desirable feature for processing of pharmaceutical formulations as the shelf-life of hydrolysis sensitive compounds can be increased by the dry state of an aerogel. Based on the results, it can be concluded that the freeze-drying did not significantly affect the drug release properties from reconstructed ANFC hydrogels. Possible structural changes in the nano-scale dimensions of ANFC fibers during freeze-drying did not affect the quality on ANFC as a hydrogel matrix platform for controlled drug delivery of small molecules and large proteins. The potential of ANFC hydrogels for controlled drug delivery applications at high fiber concentrations of $3 \%$ and $6.5 \%$ has been clearly demonstrated.

The ANFC fiber concentration and the high surface area of the individual ANFC fibers can be used to control the release rate of small as well as large molecule size pharmaceutical compounds. The ANFC hydrogel concentration affected the diffusion of small molecules moderately. The underlying release mechanism of diffusion for small molecules was mainly affected by the availability of free water molecules present in the ANFC hydrogels. Diffusivities of large proteins were decreased with increasing ANFC fiber content in the hydrogels providing significant control for release kinetics. For large protein molecules, cationic charge of the molecule may be more significant factor than size with regard to the diffusion through ANFC hydrogel. The results indicate that ANFC is capable for controlled delivery of several types of molecules. Furthermore, the aerogels can be easily rehydrated and administered upon need.

\section{Acknowledgements}

The financial support from Academy of Finland (Grant no. 264988 and 258114) is gratefully acknowledged. Orion Foundation of the Professor pool, Finland is greatly acknowledged by M.Y. This project has been supported by Tekes-Industry funded GrowDex III-project.

\section{References}


Alexandrescu, L., Syverud, K., Gatti, A., Chinga-Carrasco, G., 2013. Cytotoxicity tests of cellulose nanofibril-based structures. Cellulose, 20, 1765-1775.

Aulin, C., Netrval, J., Wågberg, L., Lindström, T., 2010. Aerogels from nanofibrillated cellulose with tunable oleophobicity. Soft Matter, 6, 3298-3305.

Avdeef, A., Berger, C.M., Brownell, C., 2000. pH-metric solubility. 2: correlation between the acidbase titration and the saturation shake-flask solubility-pH methods. Pharm. Res., 17, 85-89.

Avdeef, A., Berger, C.M., 2001. pH-metric solubility: 3. Dissolution titration template method for solubility determination. Eur. J. Pharm. Sci., 14, 281-291.

Bhattacharya, M., Malinen, M.M., Lauren, P., Lou, Y., Kuisma, S.W., Kanninen, L., Lille, M., Corlu, A., GuGuen-Guillouzo, C., Ikkala, O., Laukkanen, A., Urtti, A., Yliperttula, M., 2012. Nanofibrillar cellulose hydrogel promotes three-dimensional liver cell culture. J. Control. Release, 164, 291-298.

Borges, A.C., Eyholzer, C., Duc, F., Bourban, P., Tingaut, P., Zimmermann, T., Pioletti, D.P., Månson, J.E., 2011. Nanofibrillated cellulose composite hydrogel for the replacement of the nucleus pulposus. Acta Biomater., 7, 3412-3421.

Bradford, M.M., 1976. A rapid and sensitive method for the quantitation of microgram quantities of protein utilizing the principle of protein-dye binding. Anal. Biochem., 72, 248-254.

Carpenter, J., Prestrelski, S., Arakawa, T., 1993. Separation of freezing-and drying-induced denaturation of lyophilized proteins using stress-specific stabilization: I. Enzyme activity and calorimetric studies. Arch. Biochem. Biophys., 303, 456-464.

Carpenter, J.F., Pikal, M.J., Chang, B.S., Randolph, T.W., 1997. Rational design of stable lyophilized protein formulations: some practical advice. Pharm. Res., 14, 969-975.

Censi, R., Di Martino, P., Vermonden, T., Hennink, W.E., 2012. Hydrogels for protein delivery in tissue engineering. J. Control. Release, 161, 680-692.

Chang, L.L., Shepherd, D., Sun, J., Ouellette, D., Grant, K.L., Tang, X.C., Pikal, M.J., 2005. Mechanism of protein stabilization by sugars during freeze-drying and storage: Native structure preservation, specific interaction, and/or immobilization in a glassy matrix? J. Pharm. Sci., 94, 14271444.

Chinga-Carrasco, G., Syverud, K., 2014. Pretreatment-dependent surface chemistry of wood nanocellulose for pH-sensitive hydrogels. J. Biomater. Appl., 29, 423-432.

Christensen, D., Foged, C., Rosenkrands, I., Nielsen, H.M., Andersen, P., Agger, E.M., 2007. Trehalose preserves DDA/TDB liposomes and their adjuvant effect during freeze-drying. Biochim. Biophys. Acta, 1768, 2120-2129.

Crowe, J.H., Tablin, F., Wolkers, W.F., Gousset, K., Tsvetkova, N.M., Ricker, J., 2003. Stabilization of membranes in human platelets freeze-dried with trehalose. Chem. Phys. Lipids, 122, 41-52.

Dimić-Mišić, K., Sanavane, Y., Paltakari, J., Maloney, T., 2013. Small scale rheological observation of high consistency nanofibrillar material based furnishes. Journal of Applied Engineering Science, $11,145-151$.

Diniz, J.F., Gil, M., Castro, J., 2004. Hornification-its origin and interpretation in wood pulps. Wood Sci. Technol., 37, 489-494.

Ernst, O., Zor, T., 2010. Linearization of the bradford protein assay. J. Vis. Exp., 12, e1918. 
Franks, F., 1998. Freeze-drying of bioproducts: putting principles into practice. Eur. J. Pharm. Biopharm., 45, 221-229.

Fritz, M., Radmacher, M., Cleveland, J.P., Allersma, M.W., Stewart, R.J., Gieselmann, R., Janmey, P., Schmidt, C.F., Hansma, P.K., 1995. Imaging globular and filamentous proteins in physiological buffer solutions with tapping mode atomic force microscopy. Langmuir, 11, 3529-3535.

Gilányi, T., Varga, I., Gilányi, M., Mészáros, R., 2006. Adsorption of poly(ethylene oxide) at the air/water interface: A dynamic and static surface tension study. J Colloid Interface Sci., 301, 428435.

González-González, M., Mayolo-Deloisa, K., Rito-Palomares, M., Winkler, R., 2011. Colorimetric protein quantification in aqueous two-phase systems. Process Biochem., 46, 413-417.

Gupta, P., Vermani, K., Garg, S., 2002. Hydrogels: from controlled release to pH-responsive drug delivery. Drug Discov. Today, 7, 569-579.

Hakkarainen, T., Koivuniemi, R., Kosonen, M., Escobedo-Lucea, C., Sanz-Garcia, A., Vuola, J., Valtonen, J., Tammela, P., Mäkitie, A., Luukko, K., Yliperttula, M., Kavola, H., 2016. Nanofibrillar cellulose wound dressing in skin graft donor site treatment. J. Control. Release, 244, 292-301.

Han, Y., Quan, G.B., Liu, X.Z., Ma, E.P., Liu, A., Jin, P., Cao, W., 2005. Improved preservation of human red blood cells by lyophilization. Cryobiology, 51, 152-164.

Hannukainen, K., Suhonen, S., Savolainen, K., Norppa, H., 2012. Genotoxicity of nanofibrillated cellulose in vitro as measured by enzyme comet assay. Toxicol. Lett., 211, S71.

Hazel, J.R., Sidell, B.D., 1987. A method for the determination of diffusion coefficients for small molecules in aqueous solution. Anal. Biochem., 166, 335-341.

Higuchi, T., 1963. Mechanism of sustained-action medication. Theoretical analysis of rate of release of solid drugs dispersed in solid matrices. J. Pharm. Sci., 52, 1145-1149.

Higuchi, T., 1961. Rate of release of medicaments from ointment bases containing drugs in suspension. J. Pharm. Sci., 50, 874-875.

Hoffman, A.S., 2012. Hydrogels for biomedical applications. Adv. Drug Deliv. Rev., 64, Supplement 18-23.

Hua, K., Carlsson, D.O., Ålander, E., Lindström, T., Strømme, M., Mihranyan, A., Ferraz, N., 2014. Translational study between structure and biological response of nanocellulose from wood and green algae. RSC Adv., 4, 2892-2903.

Hubálek, Z., 2003. Protectants used in the cryopreservation of microorganisms. Cryobiology, 46, 205-229.

Isogai, A., Saito, T., Fukuzumi, H., 2011. TEMPO-oxidized cellulose nanofibers. Nanoscale, 3, 7185 .

Jeon, S., Lee, J., Andrade, J., De Gennes, P., 1991. Protein—surface interactions in the presence of polyethylene oxide: I. Simplified theory. J. Colloid Interface Sci., 142, 149-158.

Jiang, F., Hsieh, Y., 2014. Super water absorbing and shape memory nanocellulose aerogels from TEMPO-oxidized cellulose nanofibrils via cyclic freezing-thawing. J. Mater. Chem. A, 2, 350-359. 
Jiang, F., Hsieh, Y., 2016. Self-assembling of TEMPO Oxidized Cellulose Nanofibrils As Affected by Protonation of Surface Carboxyls and Drying Methods. ACS Sustainable Chem. Eng., 4, 1041 1049.

Jovanović, N., Bouchard, A., Hofland, G.W., Witkamp, G., Crommelin, D.J.A., Jiskoot, W., 2006. Distinct effects of sucrose and trehalose on protein stability during supercritical fluid drying and freeze-drying. Eur. J. Pharm. Sci, 27, 336-345.

Kekäläinen, K., Liimatainen, H., Illikainen, M., Maloney, T.C., Niinimäki, J., 2014. The role of hornification in the disintegration behaviour of TEMPO-oxidized bleached hardwood fibres in a highshear homogenizer. Cellulose, 21, 1163-1174.

Kim, H., Lee, Y., Yoo, H., Kim, J., Kong, H., Yoon, J., Jung, Y., Kim, Y.M., 2012. Synthesis and evaluation of sulfate conjugated metronidazole as a colon-specific prodrug of metronidazole. J. Drug Target., 20, 255-263.

Klemm, D., Kramer, F., Moritz, S., Lindström, T., Ankerfors, M., Gray, D., Dorris, A., 2011. Nanocelluloses: A new family of nature-based materials. Angewandte Chemie Int. Ed., 50, 54385466.

Kolakovic, R., Laaksonen, T., Peltonen, L., Laukkanen, A., Hirvonen, J., 2012. Spray-dried nanofibrillar cellulose microparticles for sustained drug release. Int. J. Pharm., 430, 47-55.

Kolakovic, R., Peltonen, L., Laukkanen, A., Hellman, M., Laaksonen, P., Linder, M.B., Hirvonen, J., Laaksonen, T., 2013. Evaluation of drug interactions with nanofibrillar cellulose. Eur. J. Pharm. Biopharm., 85, 1238-1244.

Koutsopoulos, S., Unsworth, L.D., Nagai, Y., Zhang, S., 2009. Controlled release of functional proteins through designer self-assembling peptide nanofiber hydrogel scaffold. Proc. Natl. Acad. Sci. U. S. A., 106, 4623-4628.

Laurén, P., Lou, Y., Raki, M., Urtti, A., Bergström, K., Yliperttula, M., 2014. Technetium-99mlabeled nanofibrillar cellulose hydrogel for in vivo drug release. Eur. J. Pharm. Sci., 65, 79-88.

Lin, N., Dufresne, A., 2014. Nanocellulose in biomedicine: Current status and future prospect. Eur. Polym. J., 59, 302-325.

Lins, R.D., Pereira, C.S., Hünenberger, P.H., 2004. Trehalose-protein interaction in aqueous solution. Proteins, 55, 177-186.

Lou, Y., Kanninen, L., Kuisma, T., Niklander, J., Noon, L.A., Burks, D., Urtti, A., Yliperttula, M., 2014. The use of nanofibrillar cellulose hydrogel as a flexible three-dimensional model to culture human pluripotent stem cells. Stem Cells Dev., 23, 380-392.

Lou, Y.R., Kanninen, L., Kaehr, B., Townson, J.L., Niklander, J., Harjumaki, R., Jeffrey Brinker, C., Yliperttula, M., 2015. Silica bioreplication preserves three-dimensional spheroid structures of human pluripotent stem cells and HepG2 cells. Sci. Rep., 5, 13635.

Malinen, M.M., Kanninen, L.K., Corlu, A., Isoniemi, H.M., Lou, Y., Yliperttula, M.L., Urtti, A.O., 2014. Differentiation of liver progenitor cell line to functional organotypic cultures in 3D nanofibrillar cellulose and hyaluronan-gelatin hydrogels. Biomaterials, 35, 5110-5121.

Mi, Y., Wood, G., Thoma, L., 2004. Cryoprotection mechanisms of polyethylene glycols on lactate dehydrogenase during freeze-thawing. AAPS J., 6, 45-54.

Missoum, K., Bras, J., Belgacem, M.N., 2012. Water redispersible dried nanofibrillated cellulose by adding sodium chloride. Biomacromolecules, 13, 4118-4125. 
Pääkkö, M., Ankerfors, M., Kosonen, H., Nykänen, A., Ahola, S., Österberg, M., Ruokolainen, J., Laine, J., Larsson, P.T., Ikkala, O., Lindström, T., 2007. Enzymatic hydrolysis combined with mechanical shearing and high-pressure homogenization for nanoscale cellulose fibrils and strong gels. Biomacromolecules, 8, 1934-1941.

Pääkkö, M., Vapaavuori, J., Silvennoinen, R., Kosonen, H., Ankerfors, M., Lindström, T., Berglund, L.A., Ikkala, O., 2008. Long and entangled native cellulose I nanofibers allow flexible aerogels and hierarchically porous templates for functionalities. Soft Matter, 4, 2492-2499.

Peng, Y., Gardner, D.J., Han, Y., 2012. Drying cellulose nanofibrils: in search of a suitable method. Cellulose, 19, 91-102.

Peng, Y., Gardner, D.J., Han, Y., Kiziltas, A., Cai, Z., Tshabalala, M.A., 2013. Influence of drying method on the material properties of nanocellulose I: thermostability and crystallinity. Cellulose, 20, 2379-2392.

Peppas, N.A., Hilt, J.Z., Khademhosseini, A., Langer, R., 2006. Hydrogels in biology and medicine: from molecular principles to bionanotechnology. Adv Mater, 18, 1345-1360.

Powell, L.C., Khan, S., Chinga-Carrasco, G., Wright, C.J., Hill, K.E., Thomas, D.W., 2016. An investigation of Pseudomonas aeruginosa biofilm growth on novel nanocellulose fibre dressings. Carbohydr. Polym., 137, 191-197.

Prestrelski, S., Arakawa, T., Carpenter, J., 1993. Separation of freezing-and drying-induced denaturation of lyophilized proteins using stress-specific stabilization: II. Structural studies using infrared spectroscopy. Arch. Biochem. Biophys., 303, 465-473.

Saito, T., Kimura, S., Nishiyama, Y., Isogai, A., 2007. Cellulose nanofibers prepared by TEMPOmediated oxidation of native cellulose. Biomacromolecules, 8, 2485-2491.

Saito, T., Nishiyama, Y., Putaux, J., Vignon, M., Isogai, A., 2006. Homogeneous suspensions of individualized microfibrils from TEMPO-catalyzed oxidation of native cellulose. Biomacromolecules, 7, 1687-1691.

Sheng, J.J., Kasim, N.A., Chandrasekharan, R., Amidon, G.L., 2006. Solubilization and dissolution of insoluble weak acid, ketoprofen: Effects of pH combined with surfactant. Eur. J. Pharm. Sci., 29, 306-314.

Siepmann, J., Peppas, N.A., 2011. Higuchi equation: derivation, applications, use and misuse. Int. J. Pharm., 418, 6-12.

Siepmann, J., Siepmann, F., 2012. Modeling of diffusion controlled drug delivery. J. Control. Release, 161, 351-362.

Sigma-Aldrich, Supplier's data.

Singhai, A.K., Jain, S., Jain, N.K., 1996. Cosolvent solubilization and formulation of an aqueous injection of ketoprofen. Pharmazie, 51, 737-740.

Slaughter, B.V., Khurshid, S.S., Fisher, O.Z., Khademhosseini, A., Peppas, N.A., 2009. Hydrogels in regenerative medicine. Adv. Mater, 21, 3307-3329.

Szymańska, A., Ślósarek, G., 2012. Light Scattering Studies of Hydration and Structural Transformations of Lysozyme. Acta Physica Polonica, A., 121, 694-698.

Tang, X.C., Pikal, M.J., 2004. Design of freeze-drying processes for pharmaceuticals: practical advice. Pharm. Res., 21, 191-200. 
Thakkar, V., Shah, P., Soni, T., Parmar, M., Gohel, M., Gandhi, T., 2009. Goodness-of-fit modeldependent approach for release kinetics of levofloxacin hemihydrates floating tablet. Dissolut. Technol., 16, 35-39.

Thérien-Aubin, H., Wang, Y., Nothdurft, K., Prince, E., Cho, S., Kumacheva, E., 2016. TemperatureResponsive Nanofibrillar Hydrogels for Cell Encapsulation. Biomacromolecules, 17, 3244-3251.

Valo, H., Arola, S., Laaksonen, P., Torkkeli, M., Peltonen, L., Linder, M.B., Serimaa, R., Kuga, S., Hirvonen, J., Laaksonen, T., 2013. Drug release from nanoparticles embedded in four different nanofibrillar cellulose aerogels. Eur. J. Pharm. Sci., 50, 69-77.

Vartiainen, J., Pöhler, T., Sirola, K., Pylkkänen, L., Alenius, H., Hokkinen, J., Tapper, U., Lahtinen, P., Kapanen, A., Putkisto, K., Hiekkataipale, P., Eronen, P., Ruokolainen, J., Laukkanen, A., 2011. Health and environmental safety aspects of friction grinding and spray drying of microfibrillated cellulose. Cellulose, 18, 775-786.

Walters, R.R., Graham, J.F., Moore, R.M., Anderson, D.J., 1984. Protein diffusion coefficient measurements by laminar flow analysis: method and applications. Anal. Biochem., 140, 190-195.

Wan, J., Wang, Y., Xiao, Q., 2010. Effects of hemicellulose removal on cellulose fiber structure and recycling characteristics of eucalyptus pulp. Bioresour. Technol., 101, 4577-4583.

Weishaupt, R., Siqueira, G., Schubert, M., Tingaut, P., Maniura-Weber, K., Zimmermann, T., ThönyMeyer, L., Faccio, G., Ihssen, J., 2015. TEMPO-oxidized nanofibrillated cellulose as a high density carrier for bioactive molecules. Biomacromolecules, 16, 3640-3650.

Wolkers, W.F., Tablin, F., Crowe, J.H., 2002. From anhydrobiosis to freeze-drying of eukaryotic cells. Comp. Biochem. Physiol. A Mol. Integr. Physiol., 131, 535-543.

Wolkers, W.F., Walker, N.J., Tablin, F., Crowe, J.H., 2001. Human Platelets Loaded with Trehalose Survive Freeze-Drying. Cryobiology, 42, 79-87.

Wu, Y., Fassihi, R., 2005. Stability of metronidazole, tetracycline $\mathrm{HCl}$ and famotidine alone and in combination. Int. J. Pharm., 290, 1-13.

Yazdanian, M., Briggs, K., Jankovsky, C., Hawi, A., 2004. The "high solubility" definition of the current FDA guidance on biopharmaceutical classification system may be too strict for acidic drugs. Pharm. Res., 21, 293-299.

Yohannes, G., Wiedmer, S.K., Elomaa, M., Jussila, M., Aseyev, V., Riekkola, M., 2010. Thermal aggregation of bovine serum albumin studied by asymmetrical flow field-flow fractionation. Anal. Chim. Acta, 675, 191-198.

Žepič, V., Fabjan, E.Š, Kasunič, M., Korošec, R.C., Hančič, A., Oven, P., Perše, L.S., Poljanšek, I., 2014. Morphological, thermal, and structural aspects of dried and redispersed nanofibrillated cellulose (NFC). Holzforschung, 68, 657-667.

Zhang, Y., Nypelö, T., Salas, C., Arboleda, J., Hoeger, I.C., Rojas, O.J., 2013. Cellulose nanofibrils. J. Renew. Mater., 1, 195-211.

Zhou, C., Jin, Y., Kenseth, J.R., Stella, M., Wehmeyer, K.R., Heineman, W.R., 2005. Rapid pKa estimation using vacuum-assisted multiplexed capillary electrophoresis (VAMCE) with ultraviolet detection. J. Pharm. Sci., 94, 576-589.

Zor, T., Selinger, Z., 1996. Linearization of the Bradford protein assay increases its sensitivity: theoretical and experimental studies. Anal. Biochem., 236, 302-308. 
